\title{
Alejandro Estrella González, Clío ante el espejo. Un socioanálisis de E. $P$. Thompson. Cádiz: Servicio de Publicaciones de la Universidad de Cádiz, Universidad Autónoma Metropolitana, Cuajimalpa, 2012, 341 págs.
}

Esta monografía pertenece a una dinastía de trabajos sociogenéticos centrados en la reconstrucción de trayectorias intelectuales individuales: los de Moreno Pestaña sobre Foucault y Jesús Ibáñez y el de Ildefonso Marqués sobre Bourdieu. ${ }^{1}$ Estas investigaciones, que recomponen a la vez la historia del campo y la historia de lo que los sociólogos vinculados al Centre de Sociologie Europénne (París), denominan el habitus, se salen del género de estudios convencionales sobre "el autor y su obra", esos análisis de corte idiográfico, ensimismados en el examen paralelo de los textos y de la vida del creador y que tienden a olvidar la condición colectiva de toda producción intelectual.

El trabajo emprendido por Alejandro Estrella tampoco se identifica con el comentario docto y erudito que pretende sacar a la luz y sistematizar la teoría implícita del autor estudiado, forzando así una sincronización intelectualista que olvida las condiciones materiales en las que tiene lugar la creación conceptual. Por otra parte, esta monografía no habría sido posible sin la existencia, desde hace ya casi una década, de una red -de la que el recensor forma parte- nacida en Cádiz y que con este libro de Alejandro Estrella (antiguo becario postdoctoral de la Universidad de Cádiz y actualmente profesor en la Universidad Autónoma Metropolitana de México) y con otros futuros libros que tendrán que llegar, encuentra sus ramificaciones al otro lado del océano.

El estudio de caso sobre E. P. Thompson sirve aquí como experimentum crucis de una nueva metodología en los estudios sobre historiografía. Este territorio, el de la historia de la historiografía, constituye el punto de partida de Alejandro Estrella. El autor, tras su largo periplo académico por Santiago de Compostela, Leeds y México DF, ha encontrado por fin un terreno propio, un método que ha hecho suyo; ni exploración de los paradigmas y de la formación de comunidades profesionales (algo que ha inspirado aquí a trabajos importantes como los de Gonzalo Pasamar, Ignacio Peiró o Carlos Barros) ni historia conceptual al estilo de Koselleck (imitada aquí, entre otros por José Luis Villacañas o Faustino Oncina). Lo que se despliega en su libro es un socioanálisis de inspiración bourdieusiana combinado creativamente con una sociología de las redes y cadenas rituales de interacción al estilo de Randall Collins. El autor ha sabido encontrar aquí, más allá de cualquier tentación dogmática, las bondades del eclecticismo, una virtud que con tanto acierto ha sabido ponderar Jean Claude Passeron en sus reflexiones epistemológicas. ${ }^{2}$

\footnotetext{
${ }^{1}$ J. L. Moreno Pestaña, En devenant Foucault. Sociogénèse d'un grand philosophe (Paris: Éditions du Croquant, 2006); J.L. Moreno Pestaña, Filosofía y Sociología en Jesús Ibáñez. Genealogía de un pensador crítico (Madrid: Siglo XXI, 2008) e I. Marqués Perales, Génesis de la Teoría Social de Pierre Bourdieu (Madrid, CIS, 2008)

2 J. C. Passeron, Le raissonnement sociologique. Un espace non poppérien de l'argumentation, Paris, Albin Michel, 2006), 552
} 
La virtud principal de esta monografía consiste en seleccionar a un agente destacado y que ha tenido consecuencias decisivas en el destino del campo historiográfico profesional; se trata de seguir las ondas expansivas del "efecto Thompson" para mostrar cómo puede tomar forma un sano ejercicio de reflexividad crítica en historia social. Con el oficio y la frialdad de un experimentado médico forense, Estrella disecciona y extrae las condiciones sociales que hicieron posible la caja de herramientas thompsoniana. Esto le permite dar cuenta de los sesgos derivados del impensado social del creador y de las consecuencias que se siguen de su falta de control. En el caso de Thompson, este déficit de autocontrol le impidió percatarse -como muy bien señala Moreno Pestaña en el prólogo- de su propia carencia de recursos filosóficos, lo que le abocó a convertirse en un fallido teórico social sin dejar de ser al mismo tiempo, un historiador sobresaliente. No se trata por tanto de un análisis completo del pensamiento thompsoniano sino de una sociogénesis del proyecto historiográfico que puso en pie, seguido hasta la publicación de su obra principal: The Making of the English Working Class (1963).

El fracaso de Thompson como teórico tuvo que ver con su impericia a la hora de expresar en conceptos una teoría que se mantuvo siempre en estado práctico o tácito esa "teoría escondida en el relato" a la que se refiere el autor en su estudio, y que tan fecunda y atinadamente orientó las explicaciones empíricas del historiador británico. Thompson forjó una antropología humanista e intelectualista desmentida en realidad por la subjetividad de carne y hueso descrita en sus relatos históricos. Esto le condujo a diversos malentendidos a la hora de encontrarse con la tradición francesa en filosofía y ciencias sociales (Althusser, Bachelard, Canguilhem, Foucault), y se deja ver en las deficiencias epistemológicas de ese arreglo de cuentas que es la obra titulada Miseria de la teoría.

El texto se vertebra en tres grandes apartados, precedidos por una introducción de carácter metodológico. En esta se pondera el valor de la "historia social de la historia social" como una herramienta para corregir los excesos intelectualistas y profesorales de la propia mirada histórica. La primera parte, titulada "Los senderos del profeta y la llamada de la historia", reconstruye minuciosamente la gestación el habitus primario de E. P. Thompson, siguiendo el proceso en el medio familiar, escolar y profesional, incluida la experiencia de la Guerra y de la militancia en el Partido Comunista. Esta trayectoria, muy marcada en el historiador por las relaciones con la herencia paterna y fraterna, es encuadrada también en el amplio contexto de la historiografía británica hasta mediados de la década de los 50, delimitando las estructuras principales de este campo científico y la entronización de Thompson en el mismo.

La segunda parte (“En busca de un valle que dé fruta”), sigue de cerca la formación del marco conceptual thompsoniano, emplazando la singladura del historiador dentro del subcampo político-intelectual que constituyó la iniciativa de New Left durante los años más recios de la Guerra Fría. Posteriormente, en uno de los momentos cumbres de esta monografía, se analiza el encuentro de E. P. Thompson con la figura y la obra de William Morris, una etapa crucial para entender el primer esbozo de los conceptos thompsonianos de "subjetividad" y de "agencia", forjados en esa experiencia combinada de Morris, de su herencia política y de las posibilidades del humanismo socialista en el horizonte de la Guerra Fría. 
La última parte del trabajo es una meticulosa reconstrucción de las condiciones sociales e intelectuales que hicieron posible la redacción del The Making of the English Working Class, sin duda una de las obras maestras de la historiografía contemporánea. Por una parte se recomponen los motivos y el público al que podía apuntar un proyecto como éste, en el que se trata de evitar, simultáneamente, las tentaciones del academicismo y del populismo. Posteriormente se diseccionan los grandes lineamientos arquitectónicos del libro y las disposiciones historiográficas y políticas que están en el trasfondo de su construcción. A partir de aquí se desbrozan las aportaciones teóricas involucradas en esta investigación empírica, enfatizando las peculiaridades de los conceptos de "sujeto" y de "clase social” movilizados por E. P. Thompson y que sentarán las bases de sus futuras creaciones como historiador. La lectura de este apartado ayuda a despejar nuestras dudas acerca de la noción de "teoría" con la que trabajaba el estudioso británico y permite comprender en sus justos términos lo que este pudo entender por "miseria de la teoría” en su diatriba contra el marxismo althusseriano. El mérito principal de este trabajo -que utiliza todas las fuentes primarias y secundarias disponibles correspondientes a E. P. Thompson- ${ }^{3}$ es haber dado forma expresa y coherente a esa teoría tácita de la acción y de la subjetividad presente en el autor de The Making. Pero el más difícil todavía consiste en haber evitado, en su tarea de reconstrucción, el recurso intelectualista que consiste en exhibir ex post facto la arquitectura conceptual thompsoniana, como si se hubiera obtenido la osamenta después de descarnar al cadáver. El empeño de Estrella se parece más en esto a la vivisección que a la autopsia. Ha rastreado en la trayectoria y en el cuerpo viviente de Thompson la génesis de su modus operandi hasta llegar a su culminación con la publicación del The Making. Ha sabido mostrar cómo las disposiciones cristalizadas en el habitus primario del hijo de un pastor anglicano se transformaron en los esquemas de actuación de un scholar, de un historiador profesional. Esto se logra enfocando a cámara lenta la travesía y las reconversiones de Thompson a través de distintos mundos sociales: político, familiar, religioso e historiográfico. A este efecto, Estrella monta su angular en doble perspectiva, atendiendo a la vez al análisis de trayectoria (en particular durante los dos primeros capítulos en los que se advierte el recorrido del habitus primario en el campo escolar y en el paso por William Morris y por su herencia) y el análisis del campo y de sus estructuras (en varios apartados del primer capítulo sobre el campo historiográfico y del posterior estudio de la recepción del The Making en el medio profesional de los historiadores).

Con un derroche de inteligencia y con una escritura casi metálica, cuya precisión nunca se coge en falta, Alejandro Estrella inaugura en este libro un nuevo modo de practicar la historia de la historiografía, sin muchos precedentes en España o fuera de este país. Las posibilidades abiertas por el método utilizado nos revelan que, en los estudios sobre historiografía, queda aún mucha tela por cortar. Esperemos que la comunidad de los estudiosos, en estos tiempos de penuria para la investigación, sepa aprender la lección.

\footnotetext{
${ }^{3}$ Como señala el autor, los fondos inéditos de Thompson, distribuidos entre las bibliotecas de la Bodleian y la de Brynmar Jones (Universidad de Hull) sólo serán plenamente accesibles, según Dorothy Thompson, en el año 2043, cuando se cumplan 50 años del fallecimiento del historiador. Hay que entender por tanto que la reconstrucción propuesta en esta monografía es una work in progress, que deberá ser revisada cuando estén disponibles esos fondos documentales
} 


\section{Francisco Vázquez García \\ Universidad de Cádiz \\ francisco.vazquez@uca.es}

Fecha de recepción: 7 de agosto de 2012

Fecha de aceptación: 5 de septiembre de 2012

Publicado: 31 de diciembre de 2012

Para citar: Francisco Vázquez García, “Alejandro Estrella González, Clío ante el espejo. Un socioanálisis de E. P. Thompson. Cádiz: Servicio de Publicaciones de la Universidad de Cádiz, Universidad Autónoma Metropolitana, Cuajimalpa, 2012, 341 págs.”, Historiografías, 4 (julio-diciembre, 2012): pp. 127-130, http://www.unizar.es/historiografias/historiografias/4/vazquez.pdf 